\title{
Recording Policryps structures in photonic crystal fibers
}

\author{
D. Poudereux ${ }^{1}$, M. Caño-García* ${ }^{1}$, D. Alj $^{2}$, R. Caputo ${ }^{2}$, C. Umeton ${ }^{2}$, M.A. Geday ${ }^{1}$, J.M. Otón ${ }^{1}$ \\ and X. Quintana ${ }^{1}$ \\ ${ }^{I}$ CEMDATIC, ETSI Telecomunicación, Universidad Politécnica de Madrid, Av. Complutense 30, 28040 Madrid, \\ Spain \\ ${ }^{2}$ Department of Physics and CNR-NANOTEC, University of Calabria, Arcavacata di Rende, 87036 Cosenza, Italy
}

Received February 08, 2017; accepted March 24, 2017; published March 31, 2017

\begin{abstract}
Policryps structures of photo-curable adhesive NOA61 and nematic liquid crystal mixture E7 have been created inside selected microchannels of photonic crystal fibers (PCF). The PCF was selectively infiltrated with the photopolymer-liquid crystal mixture for the writing of a holographic tunable grating inside specific holes of the photonic fiber. A $2 \mu \mathrm{m}$ pitch grating was successfully recorded in the PCF inner holes with and without collapsing the fiber cladding. The liquid crystal is properly aligned in both cases.
\end{abstract}

Photonic crystal fibers (PCF) customarily feature a number of holes running parallel to each other along the whole length of the fiber. These holes modify the effective refractive index of the fiber core and cladding, being effectively responsible of peculiar guiding properties of PCFs. Filling the holes, either all of them or a specific set, with any material may have dramatic effects on the PCF waveguide modal distribution [1-2]. If the filling material is electrooptically active, then tunable devices driven by external signals can be implemented. Liquid crystals (LCs), being fluid and electrically-controllable birefringent, have been selected as an obvious candidate for such fillings for a number of years.

The infiltration of photonic crystal fibers with liquid crystals has greatly evolved since the first infiltration experiments [3]. The combination of the passive properties of PCFs and the active optical properties of LCs resulted in a new kind of versatile devices, the photonic liquid crystal fibers (PLCF). PLCFs have been developed and manufactured for many applications including optical communications, signal treatment, interferometers and sensors [4-9].

On the other hand, efforts have been applied to the development of electrically switchable holographic gratings in liquid crystalline composite materials. There are two main manufacturing procedures for recording these gratings, the holographic polymer-dispersed liquid crystal (H-PDLC) and its direct competitor Policryps/Polyphems gratings [10-14]. Some time ago, an H-PDLC Bragg grating recorded inside all the holes of a PCF was reported [15]. In the present work, a Policryps structure has been recorded - to the best of our

*E-mail: manuel.c@upm.es knowledge, for the first time - into selected holes of two different PCFs. This versatile structure could become the basis of a new generation of electrically controlled tunable fiber Bragg gratings.

Policryps is the acronym of polymer-liquid crystalpolymer slices. Policryps is a switchable holographic grating formed by alternated layers of polymer and nematic LC homeotropically aligned, i.e. perpendicular to the polymer edges. Mixtures for Policryps fabrication have been previously described [16]; they are typically formed by a mixture of photocurable polymer and a nematic liquid crystal. In our case, the mixture is constituted by $73 \%$ of the commercial photo-curable optical adhesive NOA 61 (Norland) and 27\% of nematic LC E7 (Merck). To form a standard Policryps configuration, a sandwiched structure made of two parallel ITO-coated glasses with a small intermediate gap (some $\mu \mathrm{m}$ ) is fabricated, and filled with the mixture at a temperature above the LC isotropic transition (about $80^{\circ} \mathrm{C}$ in our case). Then, the sample is located onto the interference pattern of two branches of a $351 \mathrm{~nm}$ UV laser. The laser is previously split into two beams of the same power (the maximum power difference must not exceed $10 \%$ ). The sample is shone at about $10 \mathrm{~mW} / \mathrm{cm}^{2}$ for about $15 \mathrm{~min}$. Droplet formation is avoided by photocuring while keeping LC in isotropic phase and by applying a reduced light intensity, since diffusion plays an important role as theoretical models predict [9]. After the curing period the sample is slowly cooled down to room temperature. The result is a set of polymer stripes separated by almost pure LC perpendicularly oriented to them.

If the above-materials are used, an estimation of the refractive index (RI) range - hence of the tunability of the structure - can be obtained. At $1550 \mathrm{~nm}$ (the third communication window), the E7 NLC [17] shows an ordinary refractive index $n_{o}=1.51$ and an extraordinary refractive index $n_{e}=1.69$. The polymer refractive index is 1.53. With these data, the grating period required to select this wavelength can be estimated applying the Bragg diffraction formulation: 


$$
m \frac{\lambda_{0}}{n}=2 \Lambda \sin \theta
$$

where $m$ is the diffraction order, $n$ is the refractive index at the vacuum wavelength $\lambda_{0}, \Lambda$ is the grating period and $\theta$ is the incident angle.

Thus the period of a Bragg grating for the first diffraction order at $1550 \mathrm{~nm}$ should be about $\Lambda \approx 0.5 \mu \mathrm{m}$. The effective refractive index of the structure may be varied roughly between 1.52 and 1.60 , which results in a wavelength shift of about $50 \mathrm{~nm}$.

The procedure used to record a Policryps structure into a PCF has followed the same protocol described for planar structures, although the experimental conditions had to be slightly varied. Two different PCFs (Blaze Photonics) have been chosen for the infiltration, both having internal holes of two different diameters. The first one is the polarization maintaining photonic crystal fiber PM-1550-01, which has a solid core with two $4.5 \mu \mathrm{m}$ holes at either side of the fiber axis, and a cladding with five rings of $2.2 \mu \mathrm{m}$ holes. The cladding pitch between the holes is $4.4 \mu \mathrm{m}$ (Fig. 1, left). The second fiber is a hollow core photonic crystal fiber HC-1550-01 with a core diameter of $10 \mu \mathrm{m}$ and a cladding pitch of $3.8 \mu \mathrm{m}$ (Fig. 1, right).

To proceed to selective filling, the smaller holes are selectively collapsed with a fusion splicer. By optimizing the duration and the current of the applied voltaic arc it is possible to collapse relatively long fiber lengths (over $1 \mathrm{~cm}$ ). Observing the collapsed zone under a microscope, it is possible to note that, under certain conditions, the wider holes remain open while all the smaller holes are collapsed. This will be eventually useful for the visualization of the filled holes with recorded Policryps.

The fiber has to be filled inside a vacuum chamber, first by capillarity in vacuum introducing the collapsed side in the mixture and then applying an external pressure. In this way it is possible to control the length and position of the filled region, in our case about $2 \mathrm{~cm}$.
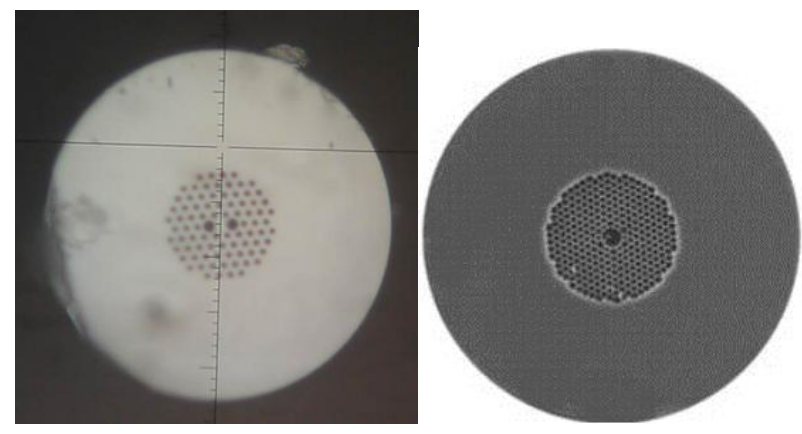

Fig. 1. PCFs employed in the experiments: PM-1550-01 (left) and HC1550 (right).
If desired, it is possible to fill the smaller holes instead. To do that, the PCF is filled with a photocurable adhesive (e.g. NOA 61). Observing under a microscope, it is easy to detect that the larger holes are filled remarkably faster than the smaller ones. Once the difference between both fronts is significant, filling is stopped, the adhesive is cured, and the fiber is cut in the region between both fronts. In this way, the smaller holes become open again while the larger holes remain obstructed by the adhesive.
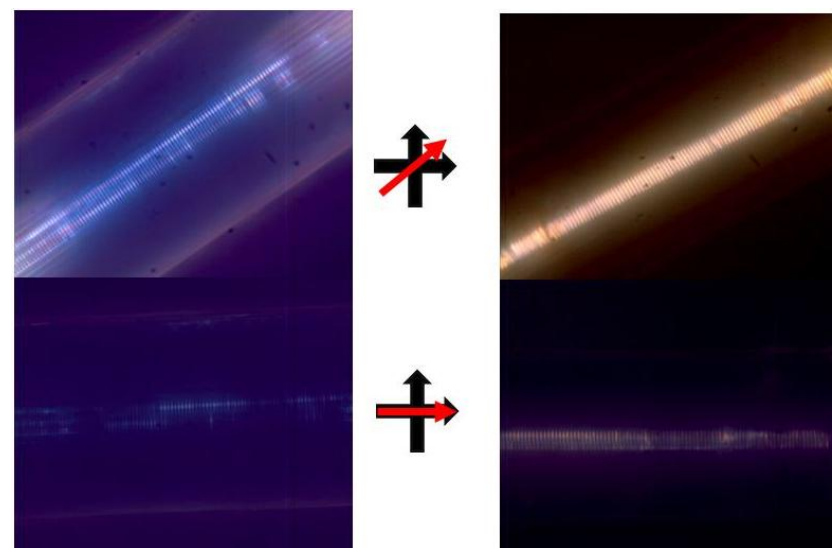

Fig. 2. Left: Microphotograph between crossed polarizers of the collapsed region of a PM-1550-01 fiber with a Policryps recorded in its wider holes, at $45^{\circ}$ (top) and parallel (bottom) to the input polarizer.

Right: Same arrangement employing HC-1550 fiber; the cladding is collapsed and a Policryps recorded in the hollow fiber core.

The resulting fiber is cut into 3 to $5 \mathrm{~cm}$ long pieces of partially filled PCF and the non-collapsed end is spliced onto a single mode fiber using a standard fusion splicer.

Images observed under the optical polarizing microscope (between crossed polarizers) reveal the formation of the grating. The pitch is $2.1 \mu \mathrm{m}$, the same that was obtained in standard Policryps fabrication in $10 \mu \mathrm{m}$ glass cells (the pitch of the grating depends on the angle between the two laser arms in the interference plane).

The $2 \mu \mathrm{m}$ pitch was chosen for this preliminary work since its manufacturing is simpler and eases microscope observation (Fig. 2). The recorded grating presents a maximum of intensity at $45^{\circ}$ between crossed polarizers as predicted in standard Policryps. When the sample is rotated by $45^{\circ}$, thus putting its axis parallel to one of the polarizers, the intensity is minimum, although it is still possible to dimly see the grating.

Pictures were taken with different contrast and exposure to reveal the faint parallel image. The maximum at $45^{\circ}$ is consistent with an alignment of the LC perpendicular to the polymer slices, and parallel to the inner surfaces of the holes.

Policryps recording of the selected holes has been confirmed in regions where the cladding was not collapsed (i.e. regions where selective filling is achieved 
while holes are collapsed elsewhere). It is worth mentioning that visualization in the microscope was more difficult in this case, due to light scattering induced by the cladding holes. Nevertheless, it was possible to observe the grating formed in the bigger holes in the interior of the fiber, as shown in Fig. 3.

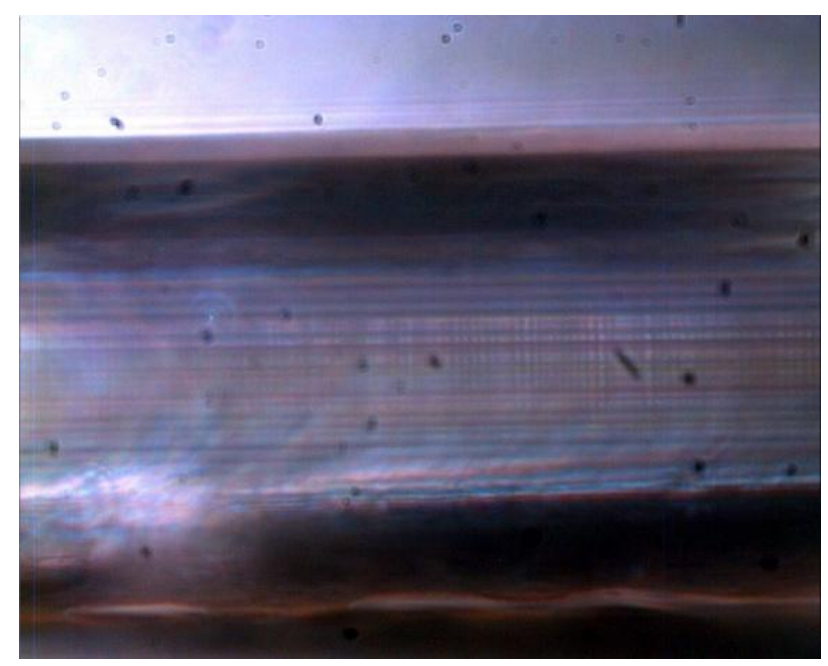

Fig. 3. Policryps recorded in the larger holes of a PM-1550-01 PCF with empty cladding as seen between crossed polarizers with the fiber parallel to one of them.

In this case, the fiber birefringence itself -produced by the smaller cladding holes acting as cylindrical lensesprecluded the visualization at $45^{\circ}$; only the $0^{\circ}$ picture is shown. It is worth stressing that the Policryps fabrication parameters must be significantly modified depending whether the smaller micro-channels are filled or empty.

Preliminar experiments of electrically induced reorientation of the LC have been performed. Although variations of light transmission have been observed between crossed polarizers, no tuning effect has been detected in this preliminar work. This is mostly due to the lack of a collimated powerful light source of an appropriate wavelength. A new set of experiments, where the grating period is set to $0.5 \mu \mathrm{m}$ (adequate for third optical window), is currently under preparation.

In conclusion, Policryps recording has been demonstrated inside the PCF holes with collapsed cladding as well as empty cladding. In this preliminary work, the pitch is too long to observe a Bragg reflection; nevertheless, the results are useful in demonstrating the realization of these structures, at least in long period gratings. In the future, shorter pitch Policryps could be achievable, thus leading to the manufacture of a switchable fiber Bragg grating. It is estimated that a pitch of $0.5 \mu \mathrm{m}$ would allow to obtain a tunability range above $50 \mathrm{~nm}$ in the $1550 \mathrm{~nm}$ region.
Authors are grateful for the financial support from Programa RETOS of the Spanish Ministerio de Economia y Competitividad (TEC2016-77242-C3-2-R AEI/FEDER, UE), the regional R\&D Program SINFOTON funded by the Comunidad de Madrid and the European Structural Funds (S2013/MIT-2790 SINFOTON-CM), and European COST Action IC1208. Manuel Caño-García is funded by the Spanish Ministerio de Economía y Competitividad, under the National Sub-Programme for Training co-funded by the European Social Fund (call 2014, ref. BES-2014-070964).

\section{References}

[1] Q. Liu, et al., J. Lightwave Technol. 34(10), 2484 (2016).

[2] L. Velázquez-Ibarra, A. Díez, E. Silvestre, M.V. Andrés, Opt. Lett. 41(11), 2600 (2016).

[3] T. Larsen, A. Bjarklev, D. Hermann, J. Broeng, Opt. Express 11(20), 2589 (2003).

[4] H.Y. Choi, M.J. Kim, B.H. Lee, Opt. Express 15(9), 5711 (2007).

[5] D. Poudereux, P. Corredera, E. Otón, J.M. Otón, X.Q. Arregui, Opt. Pura Apl. 46(4), 321 (2013).

[6] T.R. Woliński, et al., Mol. Cryst. Liq. Cryst. 619(1), 2 (2015).

[7] D. Budaszewski, T.R. Woliński, M.A. Geday, J.M. Otón, Phot. Lett. Poland, 2(3), 110 (2010).

[8] D. Alj, S. Paladugu, G. Volpe, R. Caputo, C. Umeton, Appl. Phys. Lett., 107(20), 201101 (2015).

[9] A. Veltri, R. Caputo, C. Umeton, A.V. Sukhov, Appl. Phys. Lett. 84(18), 3492 (2004).

[10] T.J. Bunning, L.V. Natarajan, V.P. Tondiglia, R.L. Sutherland, Annu. Rev. Mater. Sci. 30(1), 83 (2000).

[11] R. Caputo, L. De Sio, A.V. Sukhov, A. Veltri, C. Umeton, Opt. Lett., 29, 1261 (2004).

[12] A. Marino, F. Vita, V. Tkachenko, R. Caputo, C. Umeton, A. Veltri, G. Abbate, Euro. Phys. J. E 15, 47 (2004).

[13] G. Abbate, F. Vita, A. Marino, V. Tkachenko, S. Slussarenko, O. Sakhno, J. Stumpe, Mol. Cryst. Liq. Cryst. 453(1), 1 (2006).

[14] G. Zito, S. Pissadakis, Opt. Lett. 38(17), 3253 (2013).

[15] B. Sun, et al., IEEE Phot. Technol. Lett. 28(12), 1282 (2016).

[16] R. Caputo, et al., J. Opt. A: Pure Appl. Opt. 11(2), 024017 (2009).

[17] J. Li, S.-T. Wu, S. Brugioni, R. Meucci, S. Faetti, J. Appl. Phys. 97(7), 073501 (2005). 